\title{
High Efficiency Vertical Light Emission through a Compact Silicon Nanoantenna Array
}

\author{
Haiyang Huang ${ }^{1, \dagger}$, Hao $\mathrm{Li}^{1, \dagger}$, Wei $\mathrm{Li}^{1, \dagger}{ }^{, *}$, Aimin $\mathrm{Wu}^{1, *}$, Xin Chen ${ }^{1}$, Xuefeng Zhu ${ }^{2}$, Zhen Sheng ${ }^{1}$, \\ Shichang Zou ${ }^{1}$, Xi Wang ${ }^{1}$, Fuwan Gan ${ }^{1, *}$ \\ 1. State Key Laboratory of Functional Materials for Informatics, Shanghai Institute of Microsystem and \\ Information Technology, CAS, Shanghai 200050, China \\ 2. Department of physics, Huazhong University of Science and Technology, Wuhan 430074, China \\ † These authors contribute equally. \\ *Corresponding Author: fuwan@mail.sim.ac.cn (Fuwan Gan),wuaimin@mail.sim.ac.cn (Aimin Wu), \\ waylee@mail.sim.ac.cn (Wei Li)
}

\section{Supporting Information}

\section{Differences between the emission of the SNA and the photonic crystals leaky modes}

There are significant differences between the emission caused by a single defect in our structure and the traditional photonic crystals with leaky modes. In the traditional photonic crystals with leaky modes, the shifts of band structure are mainly due to the change of the parameters of the periodic structures such as the radius and the lattice constant. However, the defect rod is a "single" defect that has little influence on the band structure of the array in our proposed device. We calculated the 3D band structures for an ideal rod array and a defect-introduced array (identical to the structures shown in Fig.3 (a), and each cell contains eleven rods), respectively. Note that the two arrays are both finite in z-direction. The calculated 3D band structures for the two arrays are shown in Fig.S1. In this figure, there is no evidence that the corresponding Bloch wave is pushed above the light cone when the defect rod is introduced. That is to say, the role of a single defect rod always yields a defect mode in the band gap of a photonic crystal instead of changing the band, i.e., the influence of the defect on the dispersion relation in the band frequency range is negligible.

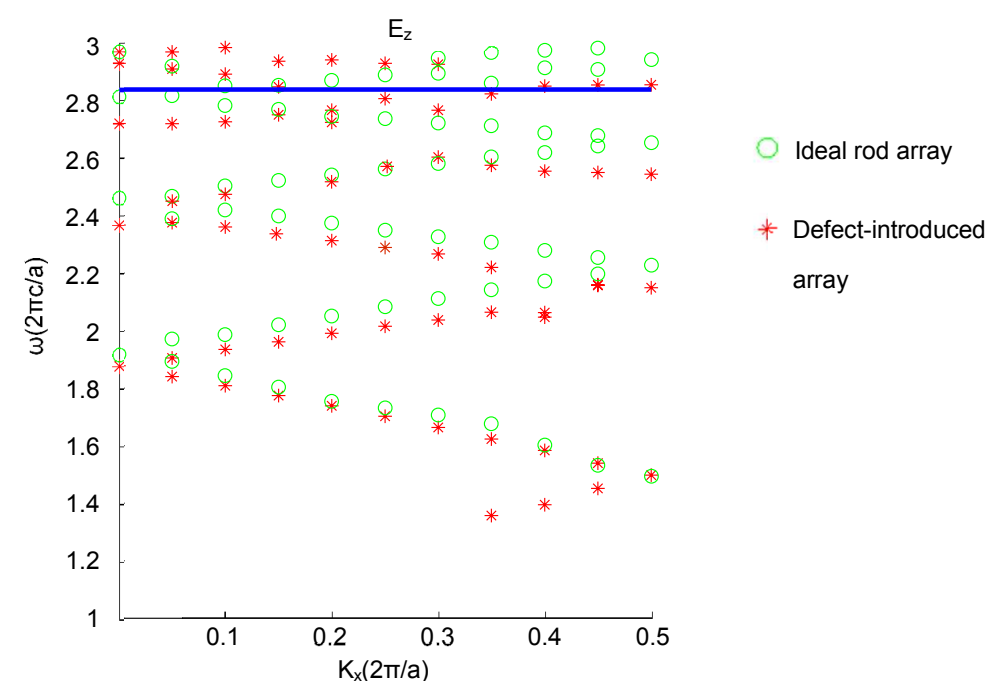


Fig.S1. Band structures for 3D rod array calculated. The superlattice contains eleven rods. All the parameters for the model struture are the same as those in the manuscript. The data shown is all above the light cone. The blue line corresponds to wavelength $1550 \mathrm{~nm}$.

\section{Field profiles at higher positions}

More simulation data with height up to $11.7 \mu \mathrm{m}$ is shown in Fig.S2. The field profile keeps on enlarging, but with some fluctuation as the height goes beyond $5 \mu \mathrm{m}$. Also, the shape of the profile gradually distorts and splits. It can be explain as: when the height increases, the relative physical dimension of the four defects becomes smaller. When the height reaches a certain level ( $5 \mu \mathrm{m}$ in our case), the four-defects area can be regard as a new single defect. Thus the emission light behaves similarly to that in Fig.3 (a), If needed, a larger SNA could be designed to have better profile at higher position. In this paper, we mainly focused on the emission distance less than $5 \mu \mathrm{m}$, because a most important application for silicon-based nanoatenna is the advanced 3D nano-packaging in silicon photonics. $5 \mu \mathrm{m}$ emission distance is adequate for chip-chip interconnection.
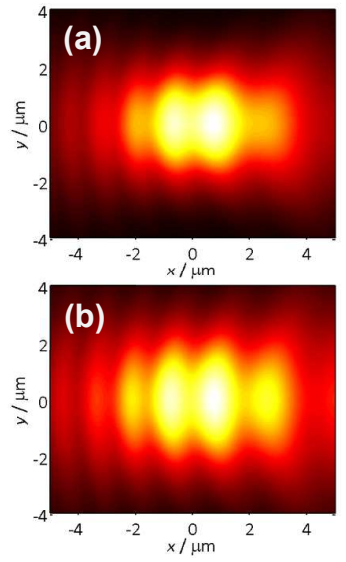
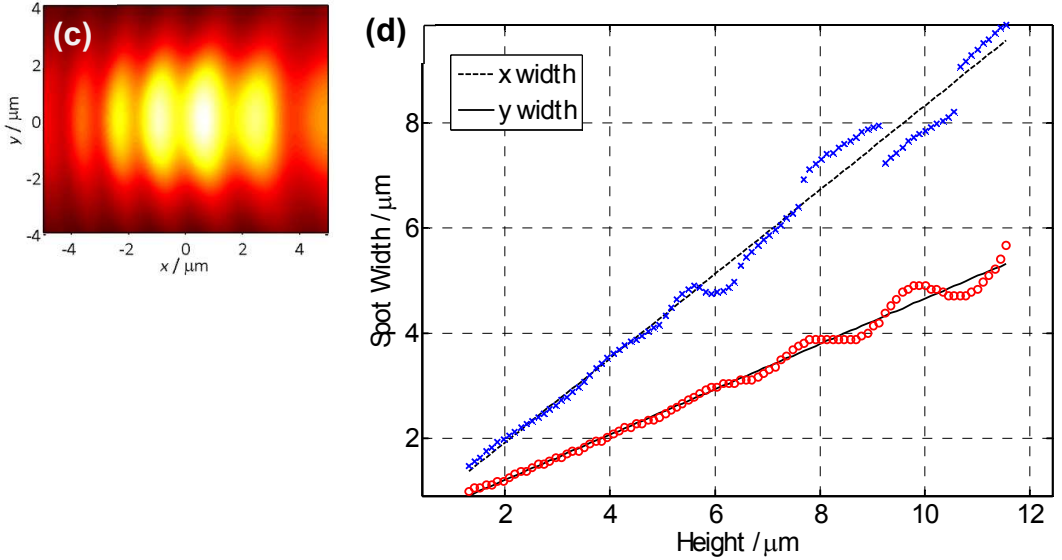

Fig.S2. Field intensity profiles at different heights are shown. (a) $7.7 \mu \mathrm{m}$, (b) $9.7 \mu \mathrm{m}$ and (c) $11.7 \mu \mathrm{m}$. Despite of some shape distortion, the profile can still be observed at $\mathrm{h}=11.5 \mu \mathrm{m}$. The corresponding plot of spot width to height (d) also shows that the quasi-linear growth (with some fluctuation) in size. 